\title{
HD 76582's Circumstellar Debris Disk
}

\author{
J. P. Marshall on behalf of the SONS consortium
}

\author{
School of Physics, UNSW Australia, High Street, Kensington, Sydney, NSW 2052, Australia \\ email: jonty.marshall@unsw.edu.au
}

\begin{abstract}
The debris disk host star HD 76582 was observed at $450 \mu \mathrm{m}$ and $850 \mu \mathrm{m}$ as part of the JCMT/SCUBA-2 debris disk legacy survey 'Sub-millimetre Observations of Nearby Stars' (SONS). The sub-millimetre data are inconsistent with a disk undergoing a steady-state collisional cascade. Combining the sub-millimetre (sub-mm) measurements with mid- and farinfrared measurements from Spitzer and Herschel, we simultaneously model the disk's thermal emission and radial extent in a self-consistent manner.
\end{abstract}

Keywords. stars:individual: HD 76582 - stars: circumstellar matter - infrared: planetary systems - sub-millimetre: planetary systems

The presence of a cool circumstellar dust disk around a mature, main-sequence star is a visible signpost of a planetary system. These disks are the remnants of asteroidal and cometary bodies broken up in mutual collisions, and are therefore known as debris disks. Debris disks are composed of rings of icy and rocky bodies ranging in size from micrometre-sized dust grains to kilometre-sized planetesimals. These structures are analogous to the Edgeworth-Kuiper belt in our solar system. Understanding these structures is fundamental to obtaining a full picture of the outcomes of the planet formation process, and the evolution of planetary systems.

Typically, debris disk host stars are identified through the detection of infrared excess above the stellar photosphere from near-infrared to sub-mm wavelengths. Surveys to date have identified disks around $20 \%$ of main-sequence stars. Interpretation of this emission is often limited by the lack of resolved imaging of the disk to constrain its architecture.

Here we present observations and preliminary interpretation of the Herschel/PACS farinfrared and JCMT/SCUBA-2 sub-mm observations of the debris disk around HD 76582, an F0 IV star at a distance of $46 \mathrm{pc}$, taken as part of the 'SONS' survey.

We have compiled data spanning optical to far-infrared wavelengths to complement the SONS sub-mm photometry. The complete SED is shown in Fig. 1. The shape of the SED, with its mid-infrared rise, broad far-infrared excess and steep sub-mm decline, precludes the adoption of a single temperature (narrow annulus) model for the disk.

The disk is seen to be extended in the Herschel far-infrared images, providing a limit on the size of the outer system. However, the angular resolution is too poor to offer any constraint on the disk's architecture, or the location of the inner edge of the debris disk. At sub-mm wavelengths the disk is detected at both $450 \mu \mathrm{m}$ and $850 \mu \mathrm{m}$, see Fig. 2 .

The steep sub-millimetre slope of HD 76582's disk is of particular interest. A powerlaw disk model requires an exponent of 7 for the grain size distribution, compared to typical values of 3-4 expected from theory. Disks with similar slopes have been observed at far-infrared wavelengths by the Herschel Open Time Key Programme 'DUNES'.

A simple three component power-law model with astrosilicate dust composition fitted to the data reproduces the observations tolerably well. The model is comprised of two 10 au-wide annuli, and a broad component lying between these two rings. The narrow annuli follow standard assumptions regarding min/max grain size, surface brightness and 


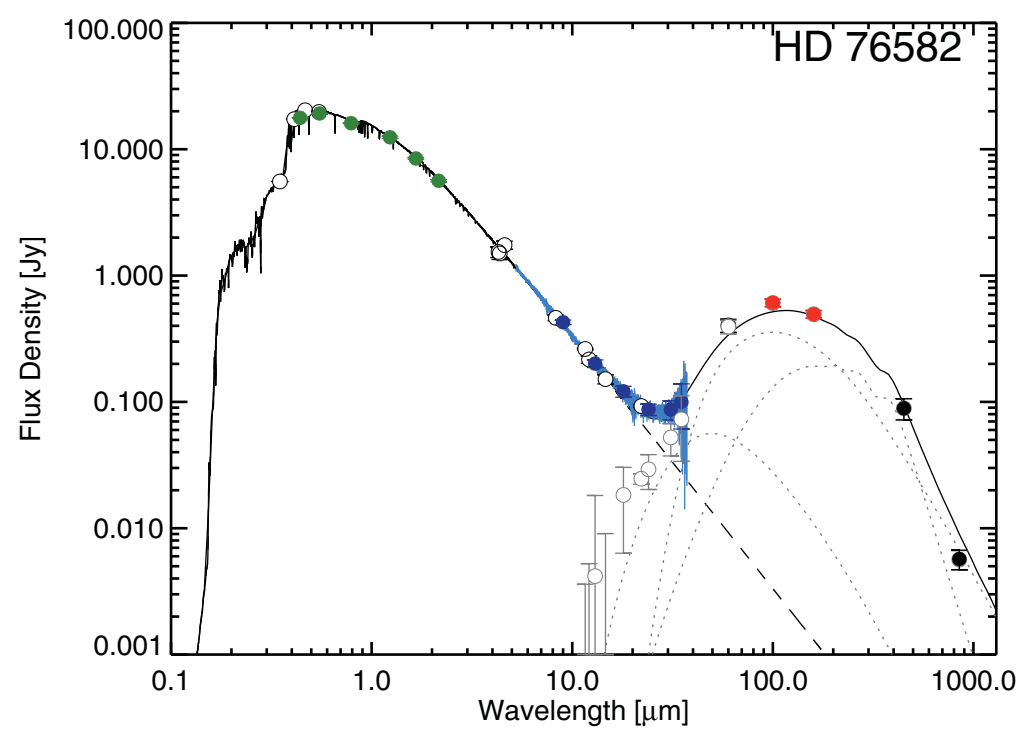

Figure 1. SED of HD 76582: green points denote optical/near-infrared photometry used to scale the photosphere model, blue points (and line) are the Spitzer/IRS data, red points are the Herschel/PACS data, black points are the JCMT/SCUBA-2 data, and the white points are other ancillary data. Excess measurements are shown as grey, hollow data points. The solid line is the total star+disk model, whilst the dashed and dotted lines denote the star and disk individual contributions, assuming a three-component disk model (see later).
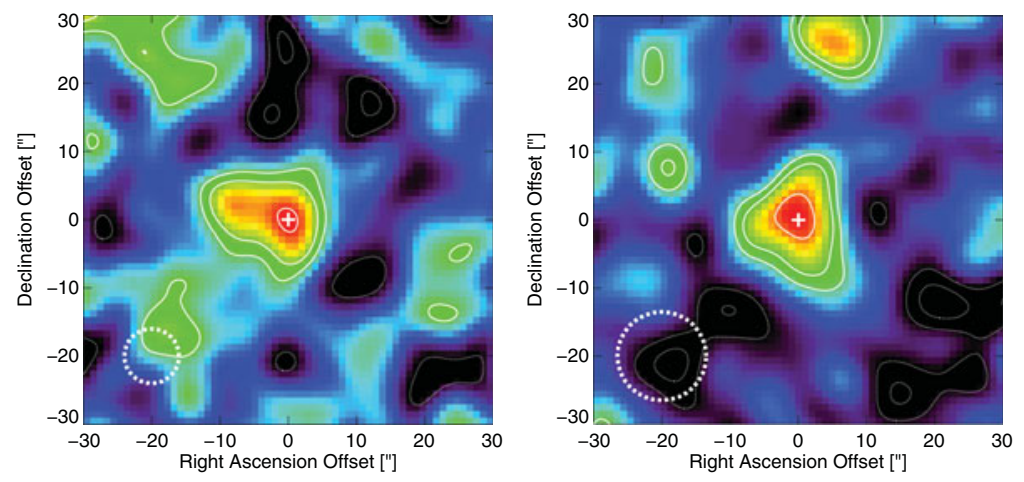

Figure 2. JCMT/SCUBA-2 images of HD 76582 at $450 \mu \mathrm{m}$ (left) and $850 \mu \mathrm{m}$ (right). The contours denote $-3,-2,2,3$, and $5-\sigma$ steps for background r.m.s. values of $0.306 \mathrm{mJy} / \operatorname{arcsec}^{2}$ at $450 \mu \mathrm{m}$ and $0.005 \mathrm{mJy} / \operatorname{arcsec}^{2}$ at $850 \mu \mathrm{m}$. Dashed contours are negative values. The white '+' denotes the stellar position. Image scale is 1 " per pixel. The instrument beam is denoted by the dashed circle in the bottom left. Orientation is north up, east left.

size distribution, but the broad component has a minimum grain size of $50 \mu \mathrm{m}$ and the steep grain size exponent mentioned previously.

Whilst a two component fit can replicate the data, it leaves significant residual flux in the images compared to the three component fit. We do not absolutely favour a three component fit however, due to the number of free parameters available rendering its adoption physically unmeaningful. 